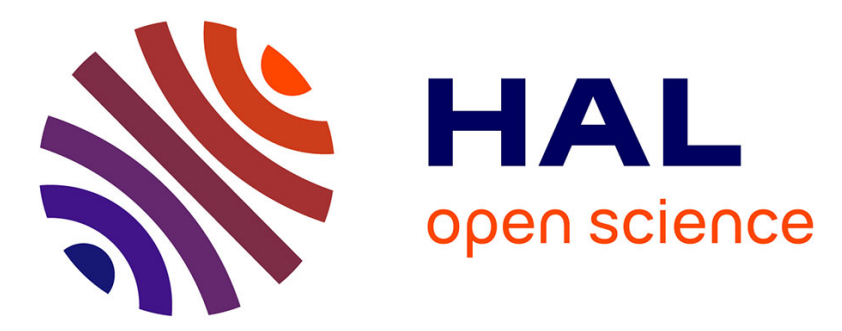

\title{
Diabatization around Conical Intersections with a New Phase-Corrected Valence-Bond-Based Compression Approach
}

\author{
Yang Zhang, Wei Wang, Benjamin Lasorne, Peifeng Su, Wei Wu
}

\section{To cite this version:}

Yang Zhang, Wei Wang, Benjamin Lasorne, Peifeng Su, Wei Wu. Diabatization around Conical Intersections with a New Phase-Corrected Valence-Bond-Based Compression Approach. Journal of Physical Chemistry Letters, 2021, 12 (7), pp.1885-1892. 10.1021/acs.jpclett.0c03506 . hal-03198676

\section{HAL Id: hal-03198676 \\ https://hal.umontpellier.fr/hal-03198676}

Submitted on 10 Nov 2021

HAL is a multi-disciplinary open access archive for the deposit and dissemination of scientific research documents, whether they are published or not. The documents may come from teaching and research institutions in France or abroad, or from public or private research centers.
L'archive ouverte pluridisciplinaire HAL, est destinée au dépôt et à la diffusion de documents scientifiques de niveau recherche, publiés ou non, émanant des établissements d'enseignement et de recherche français ou étrangers, des laboratoires publics ou privés. 


\title{
Diabatization around Conical Intersections with a New Phase- corrected Valence-Bond-Based Compression Approach
}

\author{
Yang Zhang, ${ }^{1}$ Wei Wang, ${ }^{1}$ Benjamin Lasorne, ${ }^{2 *}$ Peifeng Su, ${ }^{1 *}$ Wei $\mathrm{Wu}^{1}$ \\ ${ }^{1}$ Fujian Provincial Key Laboratory of Theoretical and Computational Chemistry, \\ The State Key Laboratory of Physical Chemistry of Solid Surfaces, and College of Chemistry \\ and Chemical Engineering, Xiamen University, Xiamen, Fujian 361005, China \\ ${ }^{2} I C G M$, Univ Montpellier, CNRS, ENSCM, Montpellier, France
}

\begin{abstract}
In the present work, the valence-bond-based compression approach for diabatization (VBCAD), previously presented in Ref. [J. Phys. Chem. Lett. 2020, 11, 5295-5301] in the case of avoided crossings, is extended to the more general situation of conical intersections and their vicinity. A pointwise phase-correction scheme for diabatic states is proposed, based on the explicit use of the peculiarities of the non-orthogonality of ab initio valence bond (VB) theory. Rather than fitting or propagating nonadiabatic couplings, it allows us to determine the phase of diabatic states consistently and automatically at each geometry point. Moreover, it is shown that the un-determination of degenerate states around a conical intersection can be fixed naturally from a straightforward classical VB picture. These are illustrated with two prototypical symmetry-induced (Jahn-Teller) conical intersection models.
\end{abstract}

${ }^{*}$ To whom correspondence should be addressed. 


\section{TOC Graphic}

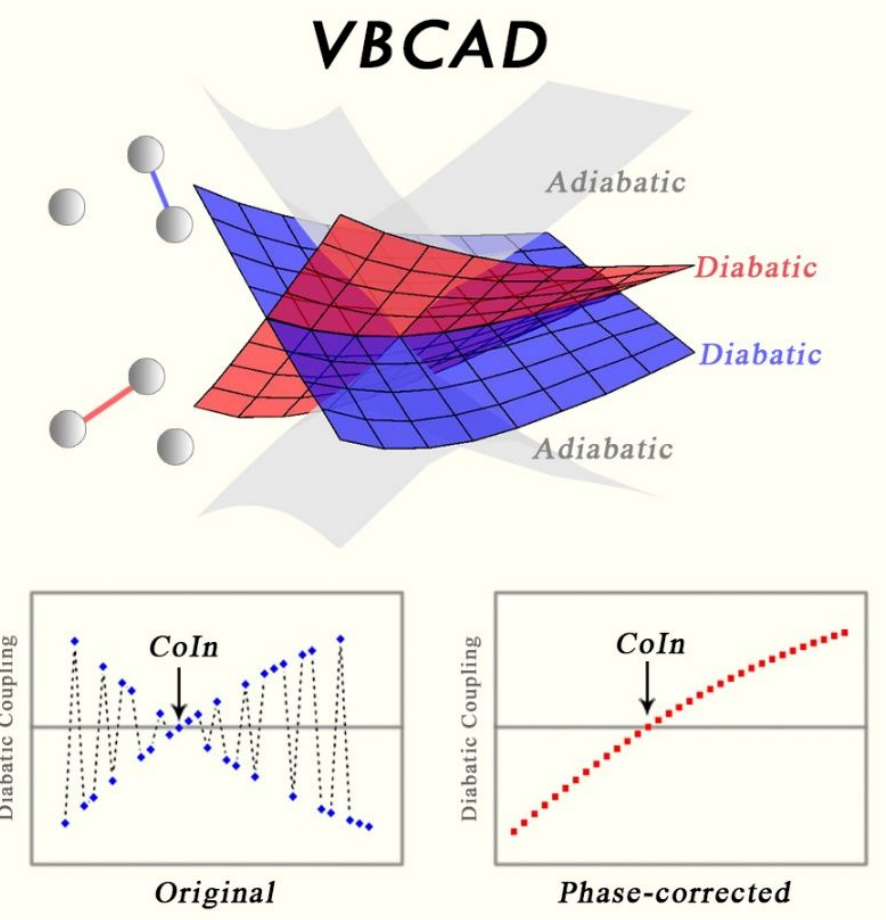


The degeneracy of electronic states leads to the breakdown of the Born-Oppenheimer approximation and thereby brings in difficulties to molecular dynamics due to the occurrence of representation singularities in the vicinity of a conical intersection. A practical cure for this is to change for a socalled diabatic representation, which formally is based on the explicit computation of non-adiabatic couplings (NAC) in the adiabatic representation and subsequent elimination of them by a convenientlydesigned unitary transformation called diabatization. In addition, if a global diabatic representation were known, we could circumvent the heavy task of calculating such couplings around conical intersections; unfortunately, such a representation is not available directly and must be constructed with some diabatization scheme, which relies on the choice of a diabatic criterion. Among them, $a b$ initio diabatization approaches are based on the properties of adiabatic electronic wave functions in terms of configurations ${ }^{1-13}$. Recently, a valence-bond-based compression approach for diabatization (VBCAD) has been proposed ${ }^{14}$; it allows a low-size diabatic Hamiltonian matrix to be built automatically. The central idea is to reduce, i.e., "compress", the full electronic Hamiltonian matrix upon employing a series of Householder transformations coupled to a VB-based diabatization criterion, which take explicit advantage of Lewis VB structures with specific bonding patterns. One of the most advantageous features of our approach is its pointwise character, which circumvents the cumbersome requirement of an integration by propagation.

However, the sign (or phase) of the diabatic wave function is a priori arbitrary in its definition and has to be initiated randomly in a pointwise diabatization approach. This issue could be fixed easily along a single coordinate associated to an avoided crossing, as we showed in our previous work, but essentially occurred as a major practical limitation of VBCAD for multidimensional situations, especially around a conical intersection. Practical solutions of such problems are obtained by 
propagating some condition that fixes the sign of diabatic wave functions or possibly with fitting procedures that minimize the (second-order) derivatives of diabatic coupling $\left(H_{12}\right)$ so as to generate a flat (or almost) $H_{12}$ surface. But these will inevitably lead to the loss of the original pointwise character of VBCAD, that is, we will increase dramatically the computational cost if we are to achieve a phase correction with such strategies, i.e., if we have to implement from a point to the next a global condition on the smoothness of the coupling functions to be fulfilled within a high-dimensional space.

In the present letter, our goal is to generalize the "VB-based diabatization by compression" approach, and thereby extend its applicability towards the vicinity of conical intersections. This will be explored according to the following aspects.

A pointwise phase-correction scheme is first proposed. In this approach, even though the nonorthogonality of the basis set has been known to bring heavy computational cost for VB calculations over the years ${ }^{15-16}$, it is shown here to become advantageous as a peculiar property that facilitates the phase-correction in the VBCAD scheme. Rather than propagating some condition here by integration, it propagates itself and its non-orthogonality smoothly by construction. That is, the phase of the diabatic wave functions as well as the sign of diabatic coupling elements can be determined automatically in a pointwise manner. This allows us to determine the phase without having to perform extra VB calculations. Moreover, the VB picture fixes the un-determination of degenerate states very naturally. Since we have equivalent choices in an MO framework because there may be several equivalent atoms due to molecular symmetry, here, one atom can be chosen specifically in a pictorial representation of $\mathrm{VB}$ wave functions via bond diagrams ${ }^{17}$. This makes it quite natural here to fix the uncertainty among degenerate cases around a conical intersection for the diabatization transformation. 
In $a b$ initio classical VB theory ${ }^{15-18}$, a many-electron wave function is expressed with a linear combination of VB structures,

$$
\Psi=\sum_{K} C_{K} \Phi_{K}
$$

where $C_{K}$ is the coefficient of the VB structure $\Phi_{K}$, which is usually defined as Heitler-London-SlaterPauling function. $\Phi_{K}$ can be further expressed as a linear combination of atomic orbital based Slater determinants,

$$
\Phi_{K}=\sum_{\kappa} d_{\kappa K} D_{\kappa}
$$

where the expansion coefficients $\left(d_{k K}\right)$ are $-1,1$ or 0 , depending on the arrangement of atomic orbitals in determinant $D_{\kappa}$. Note that various convenient extensions of this are possible in terms of basis set (partly symmetrized orbitals, breathing orbitals, etc.); however, it must be understood that VB orbitals have an intrinsic diabatic propensity by construction, which is used to its full potential here, since they vary as little as possible with the nuclear coordinates due to their local character.

The total energy and the coefficients can be obtained by solving the secular equation below:

$$
\mathbf{H C}=E \mathbf{M C}
$$

where $\mathbf{H}, \mathbf{M}$ and $\mathbf{C}$ are the VB Hamiltonian, overlap and coefficient matrices respectively. Among various classical VB methods, VBSCF ${ }^{19-20}$, which optimizes structure coefficients and orbitals simultaneously, is the elementary method of ab initio classical VB theory. If required, extra dynamic correlation can be introduced afterwards for better numerical accuracy, for example upon using the hcDFVB method ${ }^{21}$, which is a valence bond based multireference density functional theory method.

The VB-based compression approach for diabatization (VBCAD) presented here starts with the VB Hamiltonian $\mathbf{H}$ followed by a symmetric Löwdin orthogonalization ${ }^{22-23}$ yielding $\mathbf{H}^{\mathrm{L}}$. Then, a rankreduction procedure based on an eigenvalue decomposition (ED) is employed, which essentially is a 
factorization of the form,

$$
\mathbf{H}^{\mathrm{L}}=\mathbf{Q}\left(\begin{array}{cc}
\boldsymbol{\Sigma}_{\mathrm{e}} & \mathbf{0} \\
\mathbf{0} & \mathbf{0}
\end{array}\right) \mathbf{Q}^{-1}+\mathbf{Q}\left(\begin{array}{cc}
\mathbf{0} & \mathbf{0} \\
\mathbf{0} & \boldsymbol{\Sigma}_{\mathrm{n}}
\end{array}\right) \mathbf{Q}^{-1}=\mathbf{H}^{\mathrm{e}}+\mathbf{H}^{\mathrm{n}}
$$

where $\Sigma_{\mathrm{e}}$ and $\boldsymbol{\Sigma}_{\mathbf{n}}$ denote the diagonal matrices with eigenvalues $\varepsilon_{1}, \ldots, \varepsilon_{r}$ and $\varepsilon_{r+1}, \ldots, \varepsilon_{n}$ respectively, with $r$ is the number of electronic states that are required to describe the system under study over a representative range of nuclear coordinates. Note that the matrix $\mathbf{H}^{\mathrm{e}}$ is still of "full size" $n \times n$ at this stage (due to the presence of $\mathbf{Q}$ and its inverse in the similarity transform), yet of rank $r<n$ due to its congruence with $\boldsymbol{\Sigma}_{\mathbf{e}}$.

Sequentially, the symmetric matrix $\mathbf{H}^{\mathrm{e}}$ can be "compressed" into a low-dimension/size $(r \times r)$ but full-rank matrix (denoted as "pre-diabatic" $\mathbf{H}^{\text {pdia }}$ ) by a series of Householder matrix transformations (by this, we mean a block diagonalization that produces the $r \times r$ diagonal block of rank $r$ of interest together with a zero remainder diagonal block that is uncoupled). Then, the final transformation from the "pre-diabatic" Hamiltonian to the diabatic one is achieved by a two-step Householder transformation constrained by a VB-based diabatic criterion:

$$
\mathbf{U}(\alpha, \beta)=\mathbf{U}_{1}(\alpha) \mathbf{U}_{2}(\beta)
$$

This criterion was implemented in such a way that, in eq.5, $\mathbf{U}(\alpha, \beta)$ is determined by maximizing the quantity $F[\mathbf{U}(\alpha, \beta)]$ defined as:

$$
F[\mathbf{U}(\alpha, \beta)]=\sum_{K}^{n}\left|W_{K, 1}(\alpha, \beta)-W_{K, 2}(\alpha, \beta)\right|
$$

where $n$ is the number of VB structures, 1 and 2 correspond to the two diabatic states, $W_{K, i}$ is the weight of structure $K$ in the diabatic state $i$. It must be stressed here that this VB-based criterion is a way to discriminate the "optimally contracted diabatic states" according to differences in their respective compositions in terms of disjoint VB subspaces, since VB structures are diabatic by construction but there are too many to be used directly to form a practical diabatic representation. This is how and why 
VBCAD makes plain use of the VB picture from a diabatic perspective. A more detailed exposition of VBCAD has been given ${ }^{14}$.

In what follows, we present the proposed phase-correction in details. For a simple $2 \times 2$ realsymmetric Hamiltonian matrix in the diabatic representation,

$$
\mathbf{H}^{\mathrm{dia}}=\left(\begin{array}{ll}
H_{11} & H_{12} \\
H_{21} & H_{11}
\end{array}\right) ;\left(H_{12}=H_{21}\right)
$$

it is known from standard linear algebra that the two eigenvalues of $\mathbf{H}^{\text {dia }}, \varepsilon_{1}$ and $\varepsilon_{2}$, satisfy (unitary preservation of the trace and determinant),

$$
\begin{aligned}
& \varepsilon_{1}+\varepsilon_{2}=H_{11}+H_{22} \\
& \varepsilon_{1} \varepsilon_{2}=H_{11} H_{22}-\left(H_{12}\right)^{2}
\end{aligned}
$$

This means that the actual sign of $H_{12}$ from point to point is irrelevant in the formula giving the adiabatic eigenvalues $\varepsilon_{1}$ and $\varepsilon_{2}$ and thus must be fixed globally according to some consistent constraint. In this work, the central idea of the diabatic phase-correction is that the phases of the diabatic wavefunctions (represented by their expansion coefficient vectors, $\boldsymbol{C}$ in eq.1) can be determined through the geometric relationship between them. Note that the phases of the underlying VB molecular orbitals in terms of the basis set of atomic orbitals (AO) are easily determined by some constraints applied from the onset. Then, the phase correction of the $C$ expansion coefficients is achieved by enforcing the vector distance to become minimal, making plain use of the peculiarity of VB non-orthogonality. To be more specific, first, two norms based on pre-diabatic states, which can be regarded as nonorthogonal intermediate variables in the VBCAD procedure, are defined (denote as $N_{a}$ and $N_{b}$ for a two-state case) by the following formula,

$$
N_{a}=\left[\sum_{K}^{n}\left(C_{K, 1}^{\text {pdia }}-C_{K, 2}^{\text {pdia }}\right)^{2}\right]^{\frac{1}{2}} ; N_{b}=\left[\sum_{K}^{n}\left(C_{K, 1}^{\text {pdia }}+C_{K, 2}^{\text {pdia }}\right)^{2}\right]^{\frac{1}{2}}
$$

where $C_{K, i}^{\text {pdia }}$ is the coefficient of structure $K$ in the pre-diabatic state $i$. Then, the phase constraint is 
made so that if $N_{\mathrm{a}} \leq N_{\mathrm{b}}$, the signs of the pre-diabatic states are preserved,

$$
\mathbf{C}^{\text {pdia,pc }}=\mathbf{C}^{\text {pdia }} ;\left(N_{a} \leq N_{b}\right)
$$

while if $N_{\mathrm{a}}>N_{\mathrm{b}}$, the signs are corrected by a $\Sigma$ matrix,

$$
\mathbf{C}^{\text {pdia,pc }}=\mathbf{C}^{\text {pdia }} \cdot \boldsymbol{\Sigma} ; \quad\left(N_{a}>N_{b}\right)
$$

$\boldsymbol{\Sigma}$ here is defined as a diagonal matrix with dimension $n$ with 1 or -1 on its diagonal elements in order to inverse (or not) the phase of the pre-diabatic wave-functions. For a two-state case $(n=2)$,

$$
\Sigma=\left(\begin{array}{ll}
\kappa & 0 \\
0 & \lambda
\end{array}\right)
$$

where the absolute values of $\kappa$ and $\lambda$ are equal to 1 respectively but with opposite sign. It should be noted here that this "minimal vector distance" trick for phase correction works only if non-orthogonal states are employed, because $N_{\mathrm{a}}$ is identically equal to $N_{\mathrm{b}}$ if the two states are orthonormal. Furthermore, it is able to switch the constraint, i.e. if the phase correction matrix $\boldsymbol{\Sigma}$ is employed when $N_{\mathrm{a}}<N_{\mathrm{b}}$, this will make all $H_{12}$ values turned into their negative. Finally, we give a simple but intuitive geometrical illustration which helps for understanding: the angle between two non-orthogonal (but normalized) vectors is made acute by correcting the phase of either of them. Note that the whole procedure simply makes use of the preservation of the acute (or obtuse) character of the angle between both nonorthogonal vectors consistently at every point. This additional constraint is a peculiarity of VB that is turned here from an impediment to an advantage. It removes the arbitrary character of the relative phases of states, which was the only missing requirement for making the off-diagonal matrix element $H_{12}$ as smooth as possible as a function of the nuclear coordinates. The procedure of the phasecorrected VBCAD scheme is presented in the simplified flowchart displayed in Figure 1. Finally, let us stress here that the discriminating procedure is not limited in principle to a pair of states. The difference between two states is evident as a distance. For three states, this should be complemented 
with the difference between the third state and the average of the previous two, much as when building a sequential set of orthogonal Jacobi vectors from several points, so as to define a sequence of generalized relative distances. This is a group-theoretical geometrical construction that is quite general and can be found, for example in the definition of five $d$-orbitals from six monomials in a threedimensional space $(x x, x y, \ldots, z z)$. Suffice to say here that there is no formal difficulty in extending the procedure to any number of interacting states.

\section{$\underline{\text { (FIGURE } 1 \text { near here) }}$}

In a polyatomic molecule, electronic degeneracy may occur often, especially at highly symmetrical geometries. A prototypical example consists in the Jahn-Teller effect, which involves two-fold degenerate electronic states and first-order degeneracy lifting, hence stabilization upon symmetry breaking in the lower electronic state, along two-fold degenerate nuclear vibration modes. The JahnTeller effect is the simplest prototype of symmetry-induced conical intersections, which is an obvious case to start for as an illustration. Note that many molecules that possess conical intersections involved in their photochemical reactivity can be understood upon making a comparison with a Jahn-Teller analogue involving the same number of electrons ${ }^{24}$. It is known that the dissociation of $\mathrm{H}_{3}$ radical has been well studied ${ }^{25}$, in this letter, we consider the doublet triatomic hydrogen $\left(\mathbf{H}_{\mathbf{3}}\right)$ in $\mathrm{D}_{3 \mathrm{~h}}$ symmetry, which represents the smallest member of the Jahn-Teller systems. It is known that the $\mathbf{H}_{\mathbf{3}}$ system exhibits a conical intersection between the $1{ }^{2} \mathrm{~A}^{\prime}$ and $2{ }^{2} \mathrm{~A}^{\prime}$ electronic PESs for equilateral triangle geometries ${ }^{26}$. In this work, we first scanned over the bond length of all $\mathrm{D}_{3 \mathrm{~h}}$ geometries with various levels of calculation, in order to find minimum-energy $\mathrm{D}_{3 \mathrm{~h}}$ conical intersections. It is shown that the bond length is $1.38 \AA$ at the hc-DFVB/6-31G* level of theory, which provides the similar result as other MO methods ${ }^{27}$.

Figure 2. presents the double-cone shape of both potential energy surfaces (along two independent coordinates that span geometries breaking $\mathrm{D}_{3 \mathrm{~h}}$ ) as well as the curve termed the intersection 
seam (along a coordinate that spans geometries preserving $\mathrm{D}_{3 \mathrm{~h}}$ ) for the lowest-lying two adiabatic electronic states of doublet $\mathrm{H}_{3}$. They were calculated with three active electrons within three active orbitals. The state-averaged hybrid-atomic orbitals were used in VB calculations. One of the hydrogenhydrogen bonds (denoted as the H1-H3 bond) was fixed at the optimal $\mathrm{D}_{3 \mathrm{~h}}$ bond length $(1.38 \AA$ ) in the symmetry-breaking situation so as to originate first-order degeneracy-lifting distortions (branching space) from the minimum-energy conical intersection. One such variable is the distance between the midpoint $X$ of the fixed two hydrogen atoms and the third one. The other is the H2-X-H1 angle $(\theta)$, which varies from 80 to 100 degrees. As expected, the potential energy surfaces form a continuous double cone with a cusp at the equilateral triangle geometry $\left(\mathrm{D}_{3 \mathrm{~h}}\right)$.

\section{(FIGURE 2 near here)}

Figure 3a. shows a smooth crossing as expected between the diagonal diabatic matrix elements $H_{11}$ and $H_{22}$ obtained from VBCAD. They change smoothly with the variation of the nuclear geometry and cross along a curve that depends on $R$ when $\theta=90^{\circ}$. In this figure we also present the adiabatic PESs (grey surfaces). It is evident that at the crossing point, the diabatic adiabatic energies coincide. The diabatic surfaces are also tangent to the double-cone adiabatic surfaces along $\theta$ when $R$ is fixed to its value at the crossing.

\section{(FIGURE 3a,3b near here)}

Figure 3b. presents the value of the $H_{12}$ coupling matrix element along the two degeneracy-lifting coordinates (branching space). After our phase-correction was employed here, the shape of the $H_{12}$ surface becomes close to a flat plane as expected (we would obtain a folded one with a cusp line upon considering the absolute value, which could be unfolded "by hand" for a low-dimensional case such as here, but not in general without such an automated phase-correction procedure). We can see that it changes very little as a function of $\theta$ while it is essentially linear as a function of $R$ around the conical intersection, due to the choice made for the defining the two diabatic states (see below). This illustrates 
how the non-orthogonality of VB can be nicely used to get rid of the randomness of the relative phase.

Our VB-based diabatization approach relies on distinct and specifically chosen chemical bonding patterns for the diabatic representation. Figure 4. presents the chemical picture of the VB structures of the doublet $\mathbf{H}_{3}$ complex (3c-3e). There are in total eight VB structures that consist of two covalent structures and six ionic ones. Among them, $\Phi_{\text {cov1 }}, \Phi_{\text {ion1 }}$ and $\Phi_{\text {ion2 }}$ (blue marked) describe the H1-H2 two-electron bonding patterns that yield one covalent structure $\left(\Phi_{\text {cov1 }}\right)$ and two ionic ones $\left(\Phi_{\text {ion1 }}\right.$ and $\Phi_{\text {ion2 } 2) \text {; while }} \Phi_{\text {cov2 }}, \Phi_{\text {ion3 } 3}$ and $\Phi_{\text {ion4 }}$ (red marked) describe those of H2-H3 bond. This is proved by the degenerate diabatic states along the curve corresponding to the $\theta=90^{\circ}$ cut in Figure 2, where the H1$\mathbf{H} 2$ bond is equal to the $\mathbf{H 2 - H 3}$ bond in a VB picture according to the symmetry while stretching along $R$. A detailed VB weight analysis is presented in Supporting Information, which also supports this view.

\section{$\underline{\text { (FIGURE } 4 \text { near here) }}$}

Now let us turn to a second prototypical example involving, however, quite a larger number of nuclear coordinates. The cyclopentadienyl radical $\left(\mathbf{C}_{5} \mathbf{H}_{5}\right), \mathrm{Cp}$ for short, possesses a degenerate ground state with $D_{5 h}$ symmetry. It is another famous example of the Jahn-Teller effect. Much progress has been made theoretically towards understanding the implications of a conical intersection in this system $^{28-30}$. We will consider here a two-dimensional description spanned by two-fold degenerate displacements $(Q(a)$ and $Q(b))$ that will help in the description of the branching space for both adiabatic and diabatic states. To be more specific, $Q(a)$ is one of the modes that breaks $\mathrm{D}_{5 \mathrm{~h}}$ into a $\mathrm{C}_{2 \mathrm{v}}$ subgroup towards one of the ground-state minimum geometries on one side and towards the corresponding ground-state transition state (TS) geometry on the other side (there are five similar choices depending on which of the five equivalent $\mathrm{C}$ atoms is singularized). $Q(b)$ is formed as the degenerate orthogonal 
complement to $Q(a)$ and is obtained by the following formula,

$$
Q_{m}(b)=\frac{1}{2}\left(R\left(\frac{2 \pi}{5}\right) Q_{m}(a)+R\left(-\frac{2 \pi}{5}\right) Q_{-m}(a)\right)
$$

where $R$ is a geometrical rotation (within the $\mathrm{x}-\mathrm{y}$ plane) matrix through $\theta$,

$$
R(\theta)=\left(\begin{array}{ccc}
\cos \theta & -\sin \theta & 0 \\
\sin \theta & \cos \theta & 0 \\
0 & 0 & 1
\end{array}\right)
$$

The geometries were obtained at the $\operatorname{CASSCF}(5,5) / 6-31 \mathrm{G}^{*}$ level of theory. The corresponding VB calculations with an active space of five electrons into five carbon $p^{\pi}$ orbitals are denoted with $\operatorname{VBSCF}(5,5) / 6-31 \mathrm{G}^{*}$. It leads to a total set of $75 \mathrm{VB}$ structures (five covalent VB structures and seventy ionic ones) on which the different states expand as in eq.1.

\section{(FIGURE 5a, b, c near here)}

The PESs of the adiabatic ground and first excited states for $\mathrm{Cp}$ around the conical intersection are shown in Figure 5a, whereas the diabatic ones and phase-corrected coupling are depicted in Figure 5b\&c. Also, cut curves passing through the conical intersection along $Q(a)$ and $Q(b)$ are presented nearby. It is confirmed that the adiabatic PESs, which form a typical five-fold warped Mexican hat ${ }^{31}$, reflect the occurrence of a Jahn-Teller conical intersection at the central high-symmetry point $\left(\mathrm{D}_{5 \mathrm{~h}}\right)$ where $Q(a)=Q(b)$. For the diabatic ones, they exhibit a smooth crossing in the space spanned by $Q(a)$ and $Q(b)$. Similar with the $\mathrm{H}_{3}$ model presented before, the two-fold degenerate diabatic states appear along the $Q(a)$ vibration, where the diabatic coupling is a non-zero quasi-linear function of it. For $Q(b)$, the two diabatic PESs are close to the adiabatic ones and thus requires the diabatic coupling $H_{12}$ to be near zero. Note that the small curvature of the $H_{12}$ surface is expected because of the existence of quadratic terms. Furthermore, the flat (or quasi-flat) $H_{12}$ plane without cusps also proves that the automatic phase-corrected technique of VBCAD works correctly. 
It is shown that the respective natures of the diabatic states involved in VBCAD here are maintained by a relatively stable bonding pattern (specific VB structure subsets). Especially, the result of the degenerate diabatic states is converging to our chemical insights. To be more specific, the VB structure pairs, STR2 and 3 with STR4 and 5, are equivalent by symmetry along $\mathrm{C}_{2 \mathrm{~V}}$ vibrations (vertical $\mathrm{C}_{2}$ axis in Figure 6) of $Q(a)$. The detailed VB wave-function analysis is provided in Supporting Information. At last, one should stress out again here that our VB treatment fixes the un-determination of degenerate states very naturally as illustrated with the Cp example. As induced by symmetry, the rotation of the regular pentagon is allowed in principle as there exist five-fold degenerate directions. However, in the diabatic representation, this rotation in the two-dimensional branching space will mix the $H_{12}$ matrix element with diabatic energies $H_{11}$ and $H_{22}$ around the conical intersection. Thus, in the adiabatic-to-diabatic treatment within the MO framework, one should particularize one atom and make a special choice among degenerate vibrations which span the two-dimensional branching space, and the same for degenerate orbitals. Note that degenerate vibrations and orbitals are particularized randomly in practice for non-Abelian groups by quantum chemistry calculations when performing Hessian and Fock matrix diagonalizations in the situation of degenerate eigenvalues. This is determined numerically by the fact that the calculation is always made at a near-degenerate point within some numerical tolerance threshold; however, there is no direct control about the direction from this point to the truly degenerate point within the branching space, except for the history of the calculation. In contrast, in a classical VB framework, this is determined from the onset by using a particular linear independent VB structure set. Figure 6 presents a full covalent VB structure set (5 of 75 in total) of a 5-centred ring model. There exist five equivalent sets via rotations, but they share the same determinant expansion by eq.2. Thus, in order to ensure that a linearly independent basis (VB 
structure set) is employed, the degenerate Jahn-Teller prototype is fixed a priori, which means that we have already made one choice among the five equivalent sets before the classical VB calculations. This has principally become one of the advantages of VB theory in constructing diabatic representations for a high-symmetry prototype.

\section{(FIGURE 6 near here)}

In conclusion, the "VB-based compression approach for diabatization" (VBCAD) has been extended to the general and multidimensional case of a conical intersection. Rather than fitting or propagating, an automated phase-correction technique has been used and illustrated. It makes plain use of the sometimes detrimental non-orthogonality of classical VB theory. This was achieved by a phase correction matrix that makes the vector distance between two states become minimal, which thus only works for non-orthogonal cases. Our numerical results show that the a priori relative arbitrary phase of diabatic states can be made consistent from a global perspective and automatically. Moreover, the pointwise character of our approach also makes it particularly appealing for quantum or semiclassical dynamics treatments of the nuclear motion for which global and/or on-the-fly diabatic (hence smooth) potential energy and coupling surfaces are required. These advantageous features were illustrated in two prototypical symmetry-induced Jahn-Teller conical intersections: namely, the $\mathrm{H}_{3}$ and the cyclopentadienyl $\left(\mathrm{C}_{5} \mathrm{H}_{5}\right)$ radicals. Furthermore, the VB picture really fixes the un-determination of degenerate states, since chemically meaningful and nonredundant VB structures are specified from the onset and further employed. The VB wave functions obtained by VBCAD as a "black-box" also provide relevant chemical interpretation of the specific bonding patterns. These aspects obviously remind us again of the great advantages of thinking in terms of $a b$ initio valence bond theory for chemists, here in particular for constructing diabatic states on both a rational and practical basis. 
The computational details in this work are summarized as follows: The geometries were optimized with the Gaussian 16 program $^{32}$. All the VB calculations were carried out with the Xiamen Valence Bond (XMVB) program ${ }^{33}$. The VBCAD treatment was performed by a post-processing algorithm with the MATLAB program version $2018 \mathrm{~b}^{34}$. 


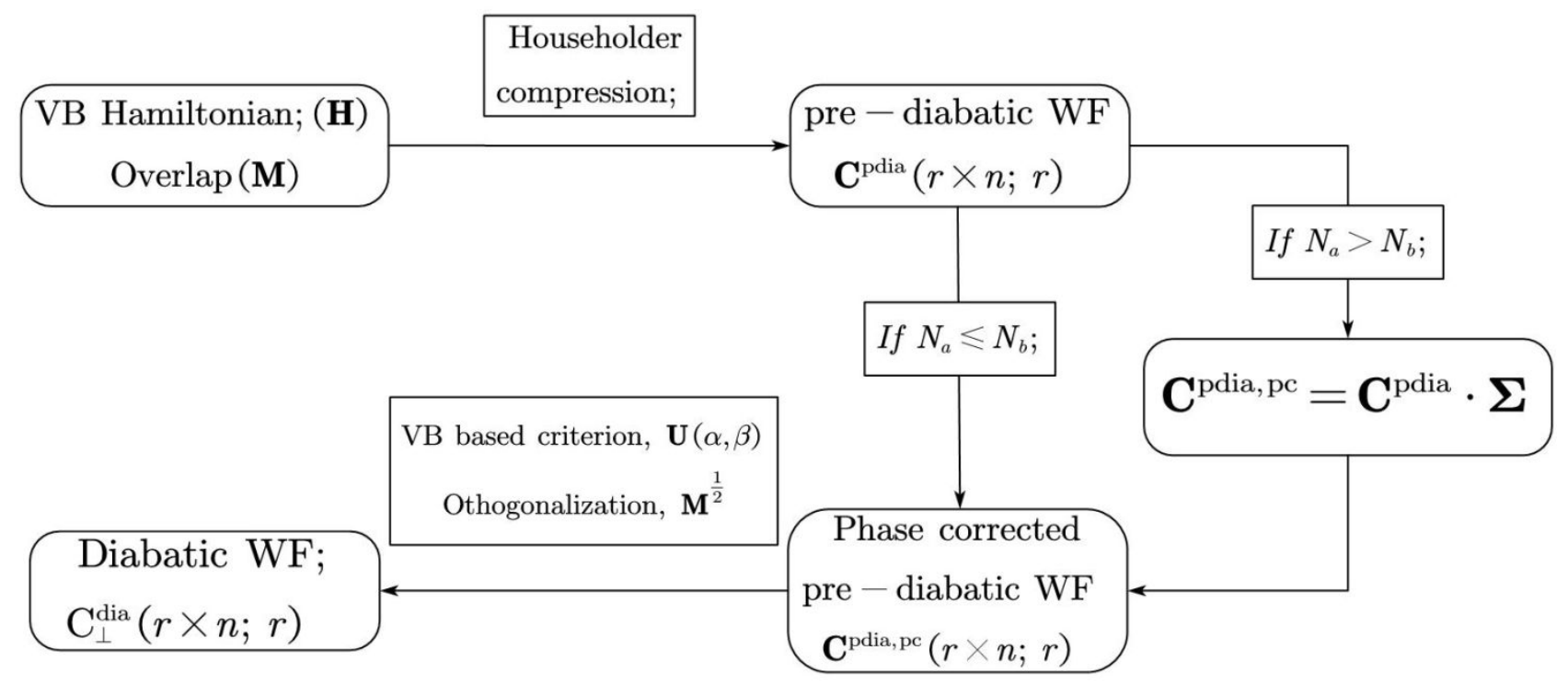

Figure 1. Simplified flowchart representing the essential steps of the phase-corrected VBCAD scheme. $\boldsymbol{N}_{\mathrm{a}}$ and $\boldsymbol{N}_{\mathrm{b}}$ denote the vector distances between two pre-diabatic states ( $\left.\mathbf{C}^{\text {pdia }}\right) . \boldsymbol{\Sigma}$ is a phase-correction matrix. 


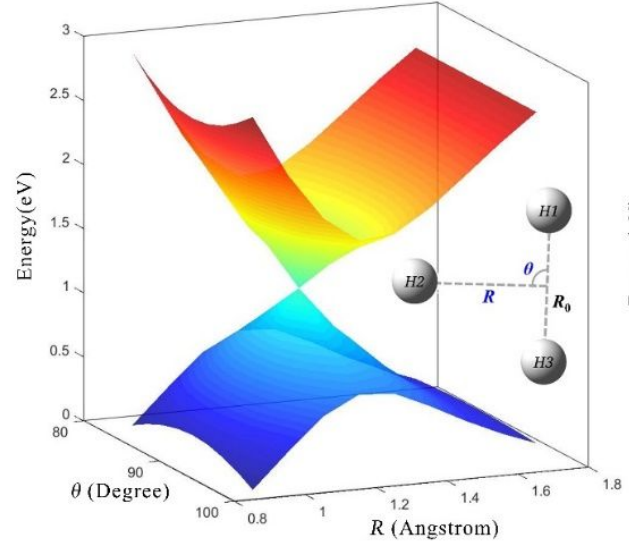

(A)

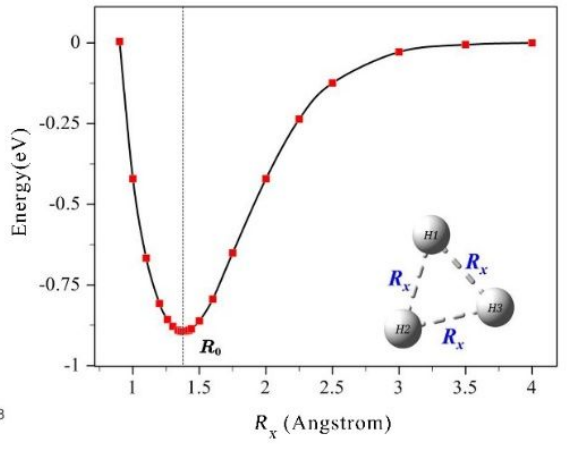

(B)

Figure 2. The global potential energy surface of the $\mathrm{H}_{3}$ radical complex in the vicinity of its conical intersection calculated by the hc-DFVB method. A) The double-cone adiabatic surfaces. $R_{0}$ is fixed at the optimal $\mathrm{D}_{3 \mathrm{~h}}$ bond length, $R$ denotes the distance between one of the atoms $(\mathrm{H} 2)$ and the midpoint $\mathrm{X}$ of the remaining two fixed $\mathrm{H}$ atoms ( $\mathrm{H} 1$ and $\mathrm{H} 3) . \theta$ denotes the $\mathrm{H} 2-\mathrm{X}-\mathrm{H} 1$ angle. $\mathrm{B})$ The degenerate ground-state and first excited-state energy curves (conical intersection seam) along a $\mathrm{D}_{3 \mathrm{~h}}$ distortion; $\mathrm{R}_{0}$ denotes the minimum $\mathrm{D}_{3 \mathrm{~h}}$ bond length $(1.38 \AA)$. 

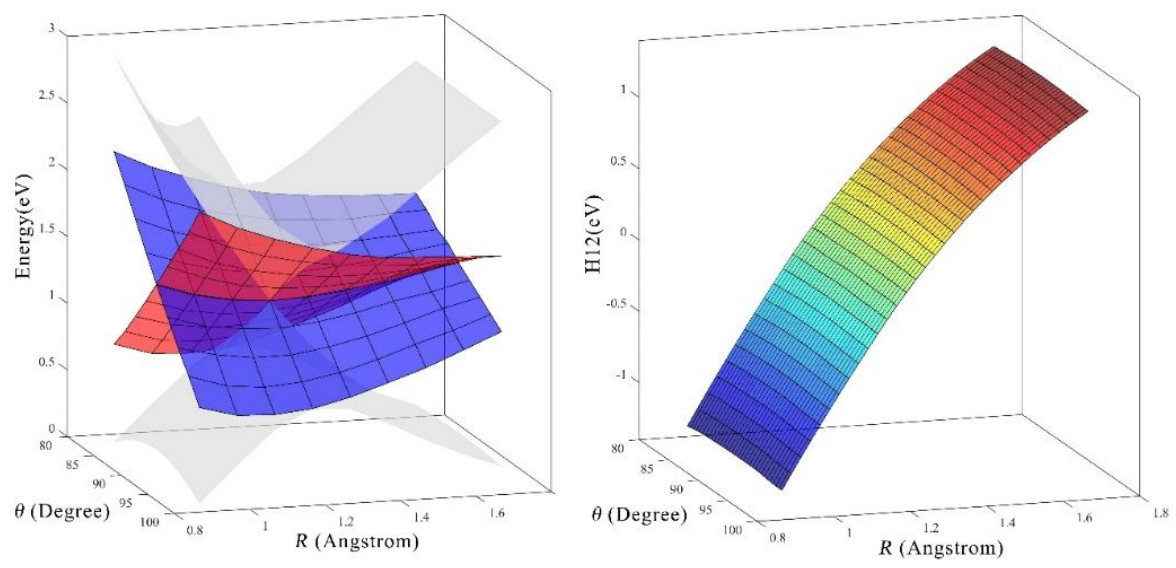

Figure 3. Energies (left panel; red and blue meshed surfaces) and coupling (right panel) of both diabatic states around the conical intersection obtained from VBCAD. The grey surfaces denote the adiabatic energies (same as in Figure 2.). 


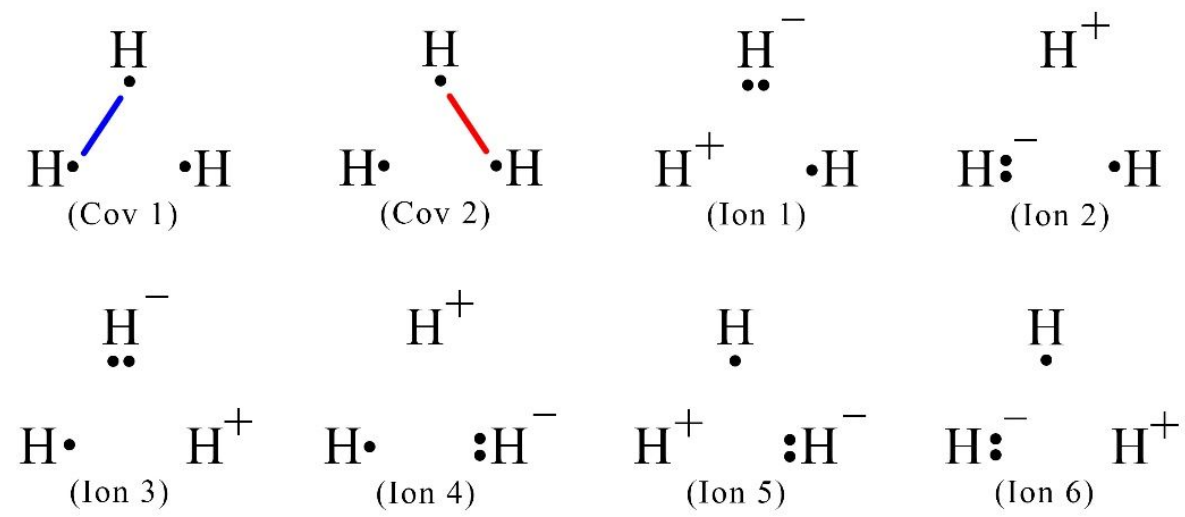

Figure 4. The linear independent VB structures of the $\mathrm{H}_{3}$ radical complex $(3 \mathrm{c}-3 \mathrm{e}) . \Phi_{\mathrm{cov} 1}$, $\Phi_{\text {ion1 } 1}$ and $\Phi_{\text {ion2 }}$ describe the H1-H2 two-electron bonding patterns that consist of one covalent structure $\left(\Phi_{\text {cov1 }}\right)$ and two ionic ones $\left(\Phi_{\text {ion } 1}\right.$ and $\left.\Phi_{\text {ion2 } 2}\right) ; \Phi_{\text {cov2 }}, \Phi_{\text {ion3 }}$ and $\Phi_{\text {ion } 4}$ describe those of the H2-H3 bond. 

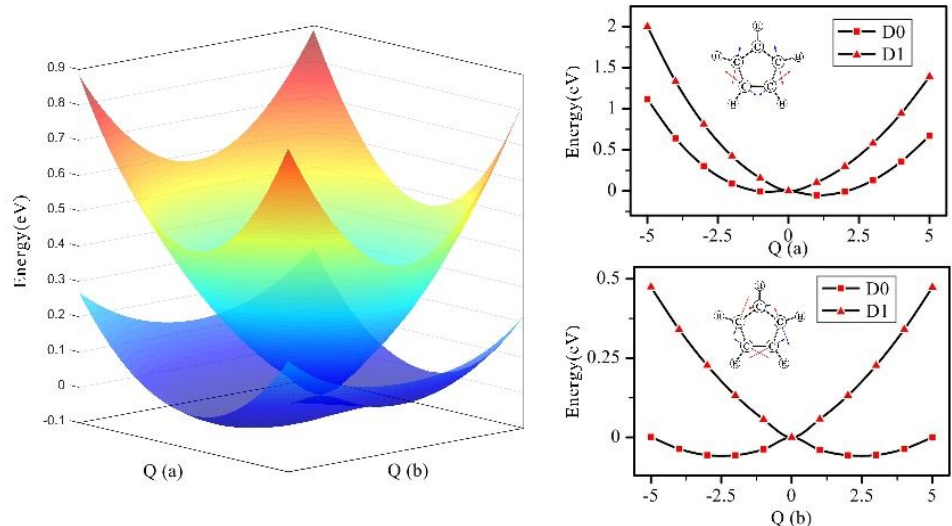

(A)
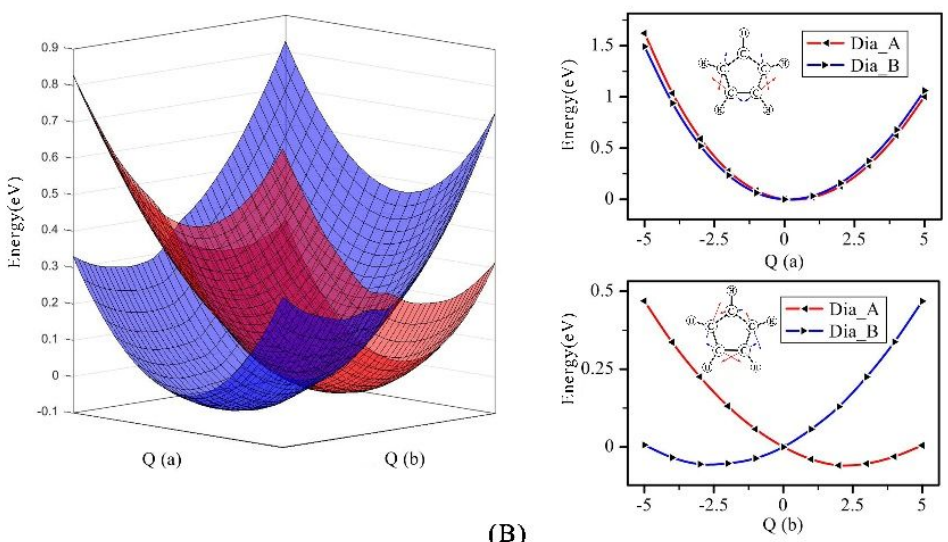

(B)
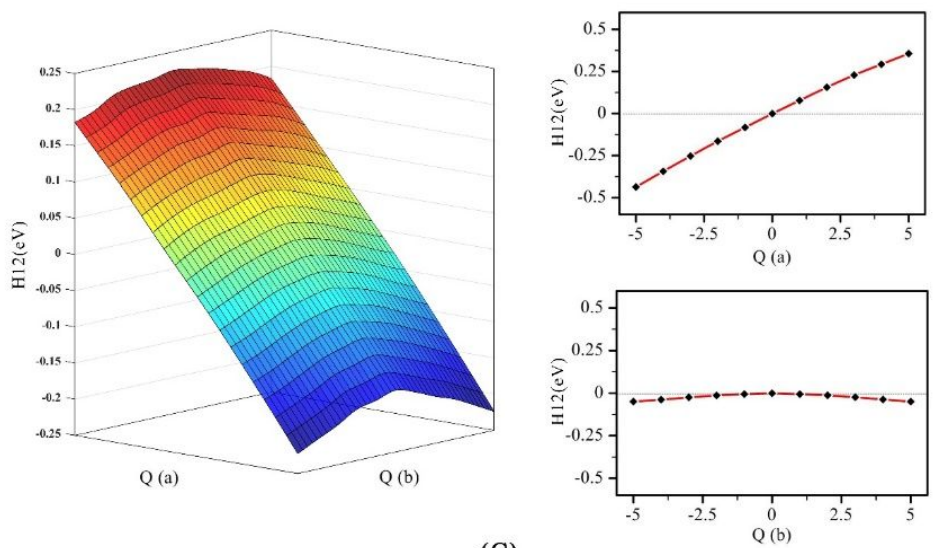

(C)

Figure 5. Potential energy surfaces of the cyclopentadienyl radical $(\mathrm{Cp})$ around the Jahn-Teller conical intersection along $Q(a)$ and $Q(b)$ distortions from its conical intersection $\left(\mathrm{D}_{5 \mathrm{~h}}\right)$ geometry. A) Adiabatic energies. B) Diabatic diagonal energies $\left(H_{11}\right.$ and $\left.H_{22}\right)$. C) Phase-corrected diabatic coupling $\left(H_{12}\right)$. 


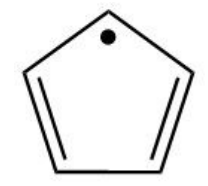

1

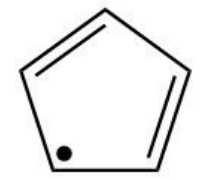

2

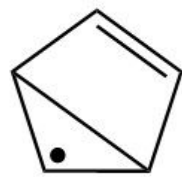

3

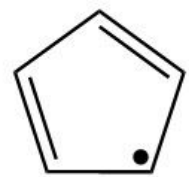

4

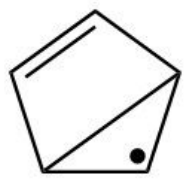

5

Figure 6. One linearly independent covalent VB structure set of $\mathrm{Cp}$ in which the upper carbon atom is particularized. The other four sets can be obtained by rotating each of the VB structure through a specific angle $(\theta ; \theta=2 n \pi / 5, n=1,2,3,4)$. 


\section{Acknowledgement:}

This project is supported by the National Natural Science Foundation of China (Nos. 21733008 and 21973077), the "111" Project (B17027), New Century Excellent Talents in Fujian Province

University and the Fundamental Research Funds for the Central Universities (No. 20720190046). 


\section{REFERENCES}

(1) Hendekovic, J. Novel Variational Definition of Diabatic States. Chem. Phys. 1982, 90, 193-197.

(2) Spiegelmann, F.; Malrieu, J. P. The Use of Effective Hamiltonians for the Treatment of Avoided Crossings. I. Adiabatic Potential Curves. J. Phys. B: At. Mol. Phys. 1984, 17, 1235-1257.

(3) Spiegelmann, F.; Malrieu, J. P. The Use of Effective Hamiltonians for the Treatment of Avoided Crossings. Ii. Nearly Diabatic Potential Curves. J. Phys. B: At. Mol. Phys. 1984, 17, 1259.

(4) Desouter-Lecomte, M.; Dehareng, D.; Lorquet, J. C. Constructing Approximatively Diabatic States from Lcao-ScfCi Calculations. J. Chem. Phys. 1987, 86, 1429-1436.

(5) Pacher, T.; Cederbaum, L. S.; Köppel, H. Approximately Diabatic States from Block Diagonalization of the Electronic Hamiltonian. J. Chem. Phys. 1988, 89, 7367-7381.

(6) Pacher, T.; Köppel, H.; Cederbaum, L. S. Quasidiabatic States from Abinitio Calculations by Block Diagonalization of the Electronic Hamiltonian: Use of Frozen Orbitals. J. Chem. Phys. 1991, 95, 6668-6680.

(7) Atchity, G. J.; Ruedenberg, K. Determination of Diabatic States through Enforcement of Configurational Uniformity. Theor. Chem. Acc. 1997, 97, 47-58.

(8) Cimiraglia, R.; Malrieu, J. P.; Persico, M.; Spiegelmann, F. Quasi-Diabatic States and Dynamical Couplings from Ab Initio Ci Calculations: A New Proposal. J. Phys. B: At. Mol. Phys. 1999, 18, 3073.

(9) Nakamura, H.; Truhlar, D. G. The Direct Calculation of Diabatic States Based on Configurational Uniformity. J. Chem. Phys. 2001, 115, 10353-10372.

(10) Grofe, A.; Qu, Z.; Truhlar, D. G.; Li, H.; Gao, J. Diabatic-at-Construction Method for Diabatic and Adiabatic Ground and Excited States Based on Multistate Density Functional Theory. J. Chem. Theory Comput. 2017, 13, 11761187.

(11) Lin, X.; Liu, X.; Ying, F.; Chen, Z.; Wu, W. Explicit Construction of Diabatic State and Its Application to the Direct Evaluation of Electronic Coupling. J. Chem. Phys. 2018, 149, 044112.

(12) Varga, Z.; Parker, K. A.; Truhlar, D. G. Direct Diabatization Based on Nonadiabatic Couplings: The N/D Method. Phys. Chem. Chem. Phys. 2018, 20, 26643-26659.

(13) Ren, M.; Ma, B.; Chen, Z.; Wu, W. Two-Dimensional Analysis of the Diabatic Transition of a General Vectorial Physical Observable Based on Adiabatic-to-Diabatic Transformation. J. Phys. Chem. Lett. 2019, 10, 5868-5872.

(14) Zhang, Y.; Su, P.; Lasorne, B.; Braïda, B.; Wu, W. A Novel Valence-Bond-Based Automatic Diabatization Method by Compression. J. Phys. Chem. Lett. 2020, 11, 5295-5301.

(15) Su, P.; Wu, W. Ab Initio Nonorthogonal Valence Bond Methods. WIREs: Comput. Mol. Sci. 2013, 3, 56-68.

(16) Chen, Z.; Wu, W. Ab Initio Valence Bond Theory: A Brief History, Recent Developments, and near Future. J. Chem. Phys. 2020, 153, 090902.

(17) Philippe, C.; Sason, S., A Chemist's Guide to Valence Bond Theory; John Wiley: Hoboken, N. J., 2007.

(18) Wu, W.; Su, P.; Shaik, S.; Hiberty, P. C. Classical Valence Bond Approach by Modern Methods. Chem. Rev. 2011, $111,7557-7593$.

(19) van Lenthe, J. H.; Balint-Kurti, G. G. The Valence-Bond Scf (Vb Scf) Method: Synopsis of Theory and Test Calculation of Oh Potential Energy Curve. Chem. Phys. Lett. 1980, 76, 138-142.

(20) van Lenthe, J. H.; Balint-Kurti, G. G. The Valence-Bond Self Consistent Field Method (Vb-Scf): Theory and Test Calculations. J. Chem. Phys. 1983, 78, 5699-5713.

(21) Zhou, C.; Zhang, Y.; Gong, X.; Ying, F.; Su, P.; Wu, W. Hamiltonian Matrix Correction Based Density Functional Valence Bond Method. J. Chem. Theory Comput. 2017, 13, 627-634.

(22) Löwdin, P. O. On the Nonorthogonality Problem Adv. Quantum Chem. 1970, 5, 185-199.

(23) Löwdin, P. O. On the Non-Orthogonality Problem Connected with the Use of Atomic Wave Functions in the 
Theory of Molecules and Crystals. J. Chem. Phys. 1950, 18, 365-375.

(24) Gatti, F.; Lasorne, B.; Meyer, H. D.; Nauts, A., Applications of Quantum Dynamics in Chemistry; Springer International Publishing, 2017; Vol. 98.

(25) Halász, G.; Vibók, Á.; Mebel, A. M.; Baer, M. A Survey Ofab Initioconical Intersections for the H+H2 System. J. Chem. Phys. 2003, 118, 3052.

(26) Porter, R. N.; Stevens, R. M.; Karplus, M. Symmetric H3: A Semiempirical and Ab Initio Study of a Simple JahnTeller System. J. Chem. Phys. 1968, 49, 5163-5178.

(27) Zhang, X.; Herbert, J. M. Analytic Derivative Couplings for Spin-Flip Configuration Interaction Singles and SpinFlip Time-Dependent Density Functional Theory. J. Chem. Phys. 2014, 141, 301-374.

(28) Yamamoto, R. N. A Casscf Study of the Cyclopentadienyl Radical: Conical Intersections and the Jahn-Teller Effect. Spectrochim. Acta A 1999, 55, 639-646.

(29) Paterson, M. J.; Bearpark, M. J.; Robb, M. A.; Blancafort, L.; Worth, G. A. Conical Intersections: A Perspective on the Computation of Spectroscopic Jahn-Teller Parameters and the Degenerate 'Intersection Space'. Phys. Chem. Chem. Phys. 2005, 7, 2100-2115.

(30) Applegate, B. E.; Barckholtz, T. A.; Miller, T. A. Explorations of Conical Intersections and Their Ramifications for Chemistry through the Jahn-Teller Effect. Chem. Soc. Rev. 2003, 32, 38-49.

(31) Domcke W., Y. D. R., Köppel H., Conical Intersections: Electronic Strucutre, Dynamics and Spectroscopy. ; World Scientific, New Jersey, 2004.

(32) Frisch, M. J.; Trucks, G. W.; Schlegel, H. B.; Scuseria, G. E.; Robb, M. A.; Cheeseman, J. R.; Scalmani, G.; Barone, V.; Petersson, G. A.; Nakatsuji, H.; et al. Gaussian 16 Rev. B.01, Wallingford, CT, 2016.

(33) Chen, Z.; Ying, F.; Chen, X.; Song, J.; Su, P.; Song, L.; Mo, Y.; Zhang, Q.; Wu, W. Xmvb 2.0: A New Version of Xiamen Valence Bond Program. Int. J. Quan. Chem. 2015, 115, 731-737.

(34) MATLAB, M. Matlab R2018b. J The MathWorks: Natick, MA, USA 2018. 